\title{
ARTÍCULOS
}

Sometido 28.10.2017. Aprobado 14.05.2018.

Evaluado por el sistema double blind review. Editor Científico: Eduardo Ayrosa

Versión original

DOI: http://dx.doi.org/10.1590/So034-759020180602

\section{CEGUERA AL PATROCINADOR: APLICACIÓN A CARTELES DE EVENTOS DEPORTIVOS}

\author{
Cegueira ao patrocinador: Utilização de banners em eventos esportivos
}

Blindness to sponsor: Application to sports event posters

\begin{abstract}
RESUMEN
El cartel deportivo es un medio de comunicación versátil, con gran repercusión y visibilidad, pero con escasa repercusión en la literatura académica. El objetivo de esta investigación es medir la atención y el recuerdo espontáneo de los patrocinadores de los carteles de eventos deportivos en función de su congruencia y emplazamiento. La muestra se segmentó de acuerdo con el sexo. El experimento con 12 estímulos de tres disciplinas deportivas revela que sí existe ceguera al patrocinador. Como predijo la teoría del emplazamiento, la posición de la marca del patrocinador generó un efecto significativo sobre la atención y el recuerdo, no habiendo diferencias significativas por sexo. Sin embargo, no encontramos diferencias por nivel de congruencia como sugiere la teoría de la transmisión de imagen. La recomendación general es situar la marca del patrocinador en la zona de acción del cartel, y aprovechar factores moderadores encontrados en la literatura como la articulación.
\end{abstract}

PALABRAS CLAVE | Patrocinio, cartel deportivo, emplazamiento, seguimiento ocular, recuerdo

\section{RESUMO}

MANUEL ALONSO DOS SANTOS ${ }^{1}$ malonso@ucsc.cl

ORCID: 0000-0001-9681-7231

\section{FERRAN CALABUIG MORENO²}

ferran.calabuig@uv.es

ORCID: 0000-0002-4538-2976

\section{MANUEL JESÚS SÁNCHEZ- FRANCO $^{3}$}

majesus@us.es

ORCID: 0000-0002-8042-3550

${ }^{1}$ Universidad Católica de la Santísima Concepción, Facultad de Ciencias Económicas y Administrativas, Concepción, Chile

${ }^{2}$ Universitat de Valencia Facultat de Ciències de l'Activitat Fisica i l'Esport, Valencia, España

3Universidad de Sevilla, Departamento de Administración de Empresas y Marketing, Sevilla, España
O banner esportivo é um meio de comunicação versátil, com grande alcance e visibilidade, mas com escassa repercussão na literatura acadêmica. O objetivo desta investigação é medir a atenção e a lembrança espontânea dos patrocinadores dos banners de eventos esportivos em função de sua congruência e localização. A amostra foi segmentada por sexo. O experimento com 12 estímulos de três modalidades esportivas revela que, sim, existe cegueira ao patrocinador. Como previa a teoria da localização, a posição da marca do patrocinador gerou um efeito significativo sobre a atenção e a lembrança, não havendo diferenças significativas por sexo, embora não tenhamos encontrado diferenças por nível de congruência como sugere a teoria da transmissão de imagem. A recomendação geral é situar a marca do patrocinador na zona de ação do banner, e aproveitar fatores moderadores encontrados na literatura como a articulação.

PALAVRAS-CHAVE / Patrocínio, banner esportivo, localização, rastreamento ocular, memória.

\section{ABSTRACT}

Advertising posters in sports sponsorship are versatile marketing tools with significant media impact and high visibility. However, the academic literature on the impact of advertising posters in this field is scarce. The purpose of this research is to measure the spontaneous recall of and attention toward business brands sponsoring sports events while assessing their location and congruence level. The sample was segmented according to gender. The experiment involved 12 stimuli related to three sporting disciplines corroborating the issue of media blindness with respect to advertising posters. Consistent with the placement theory, we found that the position of the sponsoring brand affected attention and recall with no remarkable differences between genders. Furthermore, we found no significant differences in the congruence levels, reinforcing the image transfer theory. A general guideline would be placing the sponsoring brand within the effective range of the poster while leveraging the effects of articulation and the remaining mediating variables in the literature.

KEYWORDS I Sponsorship, sports poster, site, eye tracking, memory. 


\section{INTRODUCCIÓN}

El patrocinio es una de las técnicas de marketing consideradas no convencionales que ha suscitado mayor interés en las empresas y entre los académicos en los últimos años (Papadimitriou, Kaplanidou, \& Papacharalampous, 2016); en especial el patrocinio deportivo. En esta última década está gozando de gran inversión económica e investigación científica (Meenaghan, 2013; Prendergast, Paliwal, \& Mazodier, 2016). Este creciente interés se debe, por un lado, a la creciente disminución de la eficacia de los medios convencionales $y$, por otro lado, a la internacionalización y difusión del deporte y el ocio en medios sociales (Alonso Dos Santos, 2014). La importancia económica del patrocinio es innegable. No obstante, si bien a escala mundial el gasto en patrocinio aumentó de 53.100 millones de dólares en 2013 a 62.800 millones de dólares en 2017 (IEG, 2017), los académicos aún no tienen medios consensuados para medir el retorno de la inversión (Meenaghan, 2013). Y más todavía, en el ámbito profesional aún se emplean como indicadores las apariciones netas en pantalla o en las redes sociales, la actitud hacia la marca, o el número de asistentes a los eventos, que constituyen índices ineficientes por su poca capacidad de predicción real (Breuer \& Rumpf, 2015). En consecuencia, la literatura recomienda ofrecer conocimientos, metodologías y medidas fiables y válidas para analizar, evaluar y comparar la eficacia de la actividad de patrocinio (Grohs, 2015).

En relación a la eficacia del patrocinio, las investigaciones acerca de la atención que efectivamente recibe un patrocinador es muy escasa (Breuer \& Rumpf, 2015), y específicamente en medios no televisivos (Rumpf \& Breuer, 2014). Muchas de las investigaciones publicadas emplean experimentos factoriales de imágenes impresas/digitales para contrastar la eficacia del patrocinio sin testar en primer lugar si el patrocinador realmente ha recibido atención (por ejemplo, Cornwell, Lipp, \& Purkis, 2016; Gross \& Wiedmann, 2015; Uhrich, Koenigstorfer, \& Groeppel-Klein, 2014). Otros estudios, sin embargo, realizan las encuestas en el mismo evento deportivo sin tener en consideración la atención o el número de estímulos que recibió el sujeto (Close, Lacey, \& Cornwell, 2015; Papadimitriou et al., 2016). En consecuencia, la metodología de investigación hasta ahora empleada podría estar asumiendo que los sujetos prestan la misma atención a los patrocinadores, reciben los mismos impactos y dedican el mismo tiempo a procesar la información para todos los sujetos. Pero el hecho de asumir que todos los sujetos en estudio procesan la información sobre el patrocinador es lo más preocupante. Así mismo, no podemos esperar que todos los canales de comunicación sean igual de eficaces como medio de transmisión de la imagen del patrocinador en un evento deportivo (Meenaghan \& Shipley, 1999).
La comunicación deportiva impresa ha recibido escasa atención en la literatura deportiva (Conradie, 2011; Kelly, Coote, Cornwell, \& McAlister, 2017), y el cartel deportivo es un medio muy poco explorado (Dudzik \& Gröppel-Klein, 2005). Pero su estudio es importante por varios motivos: su bajo coste, su versatilidad, su repercusión y visibilidad, y por su inversión y su popularidad (Nysveen \& Breivik, 2005). En primer lugar, es vital no suponer que la generalización de los resultados en el contexto de la publicidad televisiva, donde sí existe una profunda investigación, es aplicable a la publicidad impresa (Tipps, Berger, \& Weinberg, 2006). En la publicidad impresa deportiva la cantidad de investigaciones dedicadas es significativamente escasa. En segundo lugar, la importancia de los carteles ha sido destacada como parte fundamental de la comunicación, especialmente en eventos deportivos (Bennett, 1999). No solamente se encuentran en la calle y en instalaciones deportivas, también han trascendido el medio y se han popularizado a través de redes sociales. En tercer lugar, la comunicación por medio de cartelería impresa no es apta solamente para grandes eventos deportivos tal y como ocurre con la televisión. Los pequeños y medianos eventos deportivos también emplean este tipo de publicidad. Así pues, y teniendo en cuenta todo lo anterior, la pregunta de investigación es: ¿Qué atención recibe la marca del patrocinador de un cartel de un evento deportivo y cuál es el posterior nivel de recuerdo de dicha marca? La meta es contrastar si existe ceguera al patrocinador, su influencia sobre el recuerdo y si la atención que recibe el patrocinador depende del sexo del sujeto. A continuación, este artículo muestra las preguntas de investigación, el método experimental empleado y los resultados. Por último, se proponen las consecuencias y recomendaciones gerenciales.

\section{CEGUERA AL PATROCINADOR}

La ceguera al anuncio o ceguera al banner es un concepto que podemos trasladar al área del patrocinio para investigar la exposición y atención de los sujetos al acto de patrocinio. La ceguera al banner implica que los sujetos no reciben los estímulos procedentes del anunciante, ignoran su presencia, mientras procesan el contenido principal del estímulo (la web) (Resnick \& Albert, 2016).

El estudio de esta temática no es nuevo, en la literatura podemos encontrar varios ejemplos (Ortiz-Chaves, Martínezde-Pisón, Cancela-López-Carrión, Gonçalves-de-Vasconcellos, \& Marcos, 2014; Resnick \& Albert, 2016; Zouharová, Zouhar, \& Smutný, 2016). Las aplicaciones han sido diversas, algunos estudios se han centrado en e-commerce (Resnick \& Albert, 
2014), otros en Adwords de Google (Ortiz-Chaves et al., 2014), web 2.0 sobre turismo (Hernández-Méndez \& Muñoz-Leiva, 2015), e incluso redes sociales (Simola, Kivikangas, Kuisma, \& Krause, 2013). Las soluciones para mejorar la visibilidad de los banners que se han propuesto han sido varias (Porta, Ravarelli, \& Spaghi, 2013), por ejemplo, basadas en los componentes del mensaje (Barreto, 2013), en la estructura y tipo de información (Hsieh \& Chen, 2011), en la estética (Resnick \& Albert, 2016), o en la congruencia temática (Porta et al., 2013).

A pesar de todas las soluciones ante la ceguera al anuncio, no se ha desarrollado ni aceptado un método común y estándar de medición para concluir que existe ceguera al anuncio (Resnick \& Albert, 2016). Algunos de los estudios consultados realizan un análisis exploratorio (Muñoz-Leiva, Hernández-Méndez, Liébana-Cabanillas, \& Marchitto, 2016; Resnick \& Albert, 2014), pero la mayoría se limitan a comparar anuncios en función de su congruencia, estética o estructura (Barreto, 2013; Porta et al., 2013; Resnick \& Albert, 2016; Rieger, Bartz, \& Bente, 2015). La primera pregunta de investigación pretende examinar que existe ceguera al patrocinador, lo que implica que los usuarios no prestan atención a los patrocinadores de los carteles deportivos.

Por otro lado, el emplazamiento implícito (d'Astous \& Séguin, 1999) se refiere a la situación preferencial que la marca ocupa dentro del anuncio sin mencionarse sus características o beneficios. Según el modelo de transferencia afectiva (Liu, $\mathrm{Hu}, \&$ Grimm, 2010) basado en la teoría sobre emplazamiento (Russell, 1980), las marcas situadas en la escena de acción principal reciben mayor atención que el resto. Estos resultados son corroborados por posteriores investigaciones (Valenzuela, Martínez, \& Yáñez, 2015; Redker, Gibson, \& Zimmerman, 2013). Lo que conlleva pensar que las marcas de los patrocinadores que están emplazadas en la zona de acción recibirán más atención.

\section{RECUERDO}

Según Keller (1993) la conciencia de marca está relacionada con el recuerdo y el reconocimiento de la marca. Representa la etapa cognitiva y, generalmente, es el primer paso en los modelos jerárquicos de patrocinio. Además, representa un objetivo fundamental del patrocinio por parte de las organizaciones patrocinadoras. Los estudios de reconocimiento de marca a menudo adoptan medidas de recuerdo y reconocimiento para evaluar los niveles de sensibilización de los consumidores (Cianfrone, 2007). Según Rath y Mohapatra (2013), basándose en la teoría de la memoria, el reconocimiento de la marca es una etapa muy importante para que el consumidor construya las asociaciones de marca. Estas asociaciones, en patrocinio deportivo, son esenciales para que, según la teoría de la transferencia de imágenes, las actitudes inherentes al patrocinado se asocien con el patrocinador (Alonso Dos Santos, 2014).

Son varios los estudios de medición de los efectos del patrocinio que han incluido el reconocimiento de la marca como una dimensión para medir los niveles de conciencia de marca del consumidor (Choi \& Yoh, 2011). Siguiendo al citado autor, para medir tal dimensión se han seguido principalmente tres enfoques: (1) identificar los factores que influyen en el recuerdo del patrocinador, (2) analizar los procesos internos relacionados con el recuerdo que tiene lugar en la mente de los espectadores, y (3) medir el recuerdo de los patrocinadores.

Para medir el recuerdo se utilizan generalmente dos modos: evaluar la capacidad del consumidor para nombrar un patrocinador sin ninguna pista, o identificar al patrocinador correcto dentro de un grupo de patrocinadores potenciales o mediante un conjunto de señales. Según Wells (2000), las medidas de reconocimiento muestran interés en la promoción, mientras que las medidas de recuerdo determinan el recuerdo de la marca. Ambos métodos se utilizan en el campo del patrocinio deportivo (Cornwell, Humphreys, Maguire, Weeks, \& Tellegen, 2006; Lardinoit \& Derbaix, 2001).

Todas las formas de reconocimiento y recuerdo de marca recibidas por el consumidor directa e indirectamente a través de las actividades de patrocinio pueden influir en la decisión de compra de los consumidores mediante la creación de conciencia de marca e interés, que eventualmente puede conducir a diferencias percibidas entre la marca anunciada y otras marcas en la misma categoría de productos (Pitts \& Slattery, 2004). Por ello, las formas de medición mencionadas han sido ampliamente adoptadas por los estudiosos de marketing deportivo para evaluar la eficacia de patrocinio deportivo.

La conciencia de marca juega un papel importante en el comportamiento y en la formación de actitudes del consumidor (Keller, 1993) que puede trasladarse al consumidor de eventos deportivos principalmente por tres motivos (Lee, 2010): (1) los consumidores solo pueden evaluar la marca después de conocerla, (2) el conocimiento de marca influye en la elección de la misma, más aún en elecciones de baja implicación, y (3) el recuerdo y conocimiento de la marca condiciona al consumidor para formar una imagen de marca.

Por este motivo, se han examinado los efectos de la exposición repetida del mensaje de los patrocinadores como un mecanismo para aumentar el conocimiento de la marca o aumentar la predisposición positiva hacia dicha marca (Madrigal, 2001; Meenaghan, 2001; Pitts \& Slattery, 2004). La frecuencia de 
dicha exposición al estímulo es la variable más importante en la teoría de la mera exposición (Tom, Nelson, Srzentic, \& King, 2007); la exposición repetida afectará positivamente a los consumidores, influenciando por ejemplo, en el conocimiento y en la actitud hacia la marca patrocinadora del evento (Maxwell \& Lough, 2009), lo que ha sido aplicado anteriormente a la teoría de transferencia de imagen (Dardis, 2009). Los patrocinadores deportivos esperan que la imagen del evento deportivo se transfiera hacia su propia marca, aumentando de esta manera la intención de compra de los productos o la notoriedad de marca. Sin embargo, esta transferencia de imagen solo puede tener lugar si el consumidor reconoce o recuerda la marca que patrocina el evento deportivo. Por lo tanto, la exposición repetida al mensaje publicitario del patrocinador puede lograr un aumento de la conciencia y el recuerdo del patrocinio.

Junto con la frecuencia de exposición, la congruenciaajuste entre patrocinador y patrocinado (Papadimitriou et al., 2016; Alonso Dos Santos, Calabuig, Rejón Guardia, \& Pérez Campos, 2016; Alonso Dos Santos \& Calabuig, 2018) puede influir en el recuerdo de los patrocinadores. Según Amorim y Almeida (2015), la teoría de los esquemas y la teoría de la red asociativa (ambas teorías relacionadas con el funcionamiento de la memoria humana) facilita la recuperación de la memoria en las relaciones de patrocinio.

En la literatura de marketing podemos encontrar diversos trabajos que mencionan los beneficios del recuerdo de la marca y sus antecedentes. Así por ejemplo, Barros, Barros, Santos y Chadwick (2007) encuentran que el conocimiento previo de la marca patrocinadora refuerza el recuerdo de dicha marca. Así mismo, postulan que la preferencia por los productos del patrocinador también aumenta la probabilidad de recordar el nombre de la marca patrocinadora. Pitts y Slattery (2004) examinaron la relación entre la frecuencia de asistencia a un torneo de fútbol y el reconocimiento de la marca del patrocinador. Lee (2010), por su parte, realizó un experimento consistente en la exposición al patrocinio de un evento deportivo del NASCAR, encontrando una relación positiva y directa entre la frecuencia de la exposición al patrocinio y la actitud hacia la marca patrocinadora, lo que es consistente con la teoría de la mera exposición. Por último, Lee (2008) encuentra una relación positiva entre el recuerdo de marca y la actitud hacia el patrocinador. El recuerdo del patrocinio a menudo se ha utilizado como variable explicativa para los efectos del patrocinio (Pope \& Voges, 1999; Speed \& Thompson, 2000). Consecuentemente, podríamos asumir que la exposición al patrocinio proviene de una mayor atención a la marca del patrocinador. Entonces, una mayor fijación en tiempo y frecuencia proporcionará un mayor recuerdo de la marca.

\section{SEXO}

La relación entre el sexo de los consumidores y el marketing deportivo ha sido motivo de estudio anteriormente. Algunas investigaciones han demostrado la capacidad de la variable sexo de influir sobre el grado de identificación hacia el equipo (Menefee \& Casper, 2011), la compra de productos patrocinados (Kwon \& Armstrong, 2002), la motivación de asistencia a eventos deportivos (Funk, Toohey, \& Bruun, 2008) y la participación en actividades deportivas (Muñiz, Rodríguez, \& Suárez, 2014). Es una variable de segmentación descrita, empleada previamente en el marketing deportivo. Incluso anteriormente se ha detectado la capacidad de la segmentación por sexo de influir en el grado de recuerdo de los patrocinadores (Kinney, McDaniel, \& DeGaris, 2008), pues según McDaniel y Kinney (1999) y Stipp y Nicholas (1996) los hombres demostraron significativamente más capacidad de recuerdo que las mujeres. La interpretación de la hipótesis de la selectividad (Meyers-Levy, 1988) sugiere que las diferencias de sexo se deben a diferencias en el grado de procesamiento a nivel cognitivo, los hombres son procesadores selectivos que a menudo se basan en un subconjunto de factores disponibles y destacados en lugar de procesar mensajes detallados como las mujeres. Así mismo, se estima que la implicación de los sujetos con el patrocinado (Cornwell et al., 2006) y con el deporte influye en la atención hacia los patrocinadores (Boronczyk, Rumpf, \& Breuer, 2018), y que los hombres son los sujetos más implicados (Wann, Melnick, Russell, \& Pease, 2001). Sport fans: The psychology and et al. 2001), aficionados (Wann, Waddill, \& Dunham, 2004) e identificados con el deporte (Menefee \& Casper, 2011). Los hombres tienden a procesar la información con la que tienen un mayor grado de implicación.

Si la variable sexo se ha mostrado capaz de segmentar el grado de interacción y eficacia publicitaria (McMahan, Hovland, \& McMillan, 2009), el nivel emocional (Alonso Dos Santos \& Pérez-Campos, 2015) y la calidad y satisfacción de los eventos deportivos (Ko, Kim, Kim, Lee, \& Cattani, 2010) sería razonable pensar que puede influir sobre la atención de las comunicaciones deportivas impresas. Sin embargo, las investigaciones sobre atención al anuncio han demostrado cierta controversia. Por un lado, algunos investigadores afirman que los hombres prestan más atención a los banners que las mujeres (Goodrich, 2014), y otros investigadores afirman que no existen diferencias significativas (Barreto, 2013; Drèze \& Hussherr, 2003; MuñozLeiva et al., 2016). Por lo tanto, se espera que los hombres muestren mayores niveles de atención al patrocinador que las mujeres. 


\section{MÉTODO}

\section{Validación de los estímulos}

El proceso de construcción de los estímulos consta de varias etapas. En la primera, se seleccionan por conveniencia cuatro categorías deportivas, que son tenis, vela, remo y fórmula 1. Una muestra de 100 sujetos universitarios proporciona cuatro posibles patrocinios congruentes e incongruentes para cada una de las categorías deportivas mediante un cuestionario con preguntas abiertas.

El resultado se procesa y se seleccionan las asociaciones con mayor frecuencia. La disciplina remo fue eliminada debido al escaso número de patrocinadores asociados. El siguiente paso consiste en una encuesta con escala tipo Likert de 1 a 7 donde los sujetos deben de señalar el grado de coherencia e incoherencia de las asociaciones generadas en la primera etapa. Posteriormente, se crean las comunicaciones comerciales modificando carteles reales de eventos deportivos en los que no existan personajes reales o conocidos para evitar una posible implicación del sujeto con el personaje. La práctica habitual internacional en el diseño de cartelería deportiva, aunque no existe un guion establecido, consiste en dividir la comunicación en dos partes con un formato vertical. Dos tercios del cartel desde la parte superior contienen la información del evento y la zona de conceptualización del evento. Esta última puede consistir en la marca del evento, jugadores populares que compitan o escenas deportivas típicas de la disciplina deportiva del evento. En la zona inferior se sitúan habitualmente los patrocinadores.
Por último, un focus group permite valorar la calidad de los carteles e imágenes construidas y manipuladas. Los resultados de este test cualitativo nos permiten desechar ciertas comunicaciones donde el patrocinador o el evento no estaban bien representados o tenían una calidad de imagen insuficiente. Todas las imágenes generadas están disponibles para su descarga en los enlaces mostrados en la Tabla 1. La nomenclatura de la secuencia de observación de las imágenes contiene tres partes con la siguiente explicación: categoría deportiva (Fórmula 1 = F1, Vela $=V$, Tenis $=T$ ), congruencia $(C=$ congruente, $I=$ incongruente), y posición ( $\mathrm{D}=$ dentro de la zona de acción y $\mathrm{F}=$ fuera).

\section{El experimento}

El diseño experimental es mixto y basado en diseños previos con seguimiento ocular y banners (Hernández-Méndez \& MuñozLeiva, 2015; Muñoz-Leiva et al., 2016). Tiene un diseño intersujeto bajo las variables de sexo y disciplina, y un diseño intrasujeto mediante la posición y congruencia. Es un diseño factorial $2 \times 2$ con 12 estímulos en total divididos en tres disciplinas deportivas. Se forman cuatro grupos con tres secuencias cada uno, compuestos por diez sujetos aleatorizados y balanceado en función de la edad y el sexo del sujeto (ver Tabla 1). Pero manteniendo la asignación aleatoria de las unidades de prueba a los grupos, y los grupos al conjunto experimental congruente o no. En total, participan 120 sujetos. La Tabla 1 muestra la secuencia de observación, el nombre del grupo y el enlace de la imagen del estímulo.

Tabla 1. Secuencia de observación de los estímulos, asignación al grupo y enlace de descarga de la imagen empleada

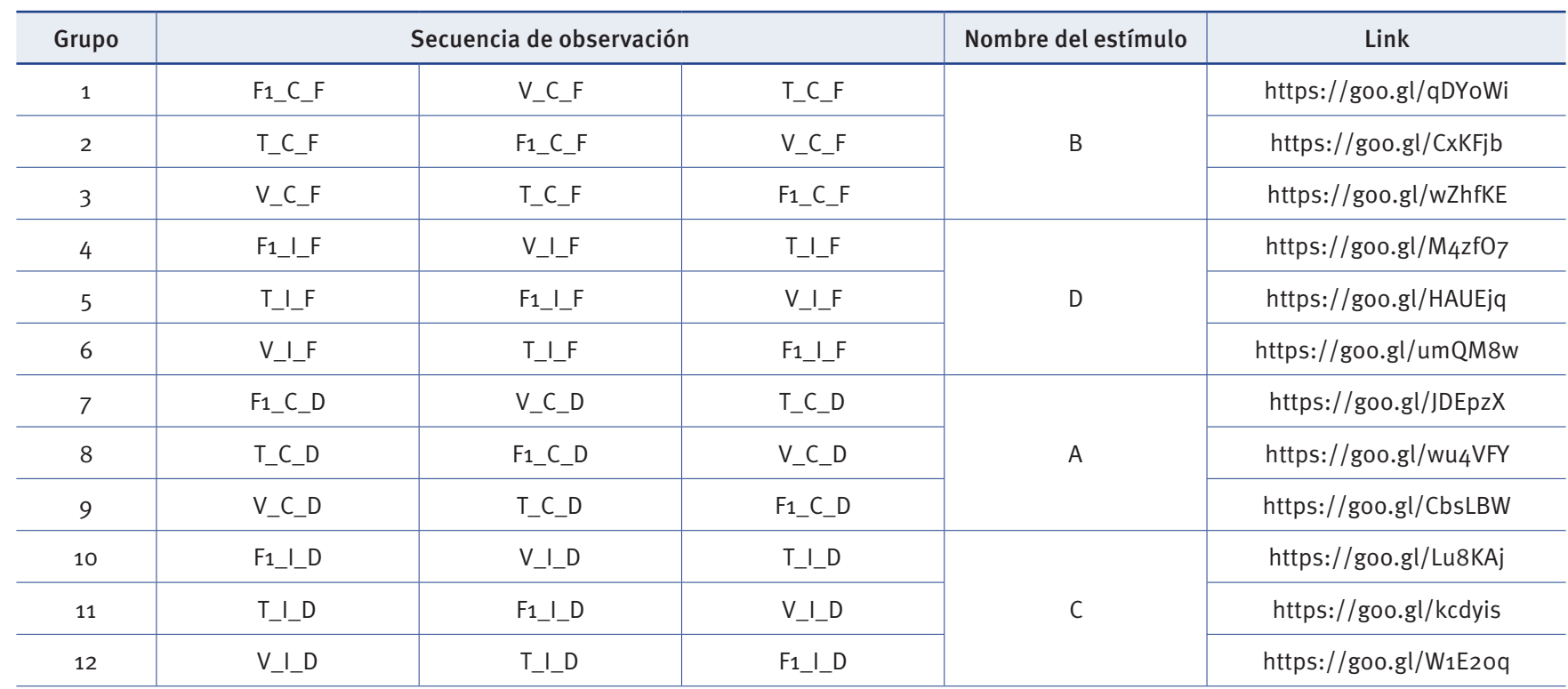


El trabajo de campo se realizó durante las primeras semanas de septiembre del año 2016 en una sala acondicionada para la experimentación neurofisiológica (International Telecommunication Union, 2002). La muestra está compuesta por 60 hombres y 60 mujeres con una edad media de 23,4 años (desviación 3,5). Es una muestra por conveniencia captada en el campus universitario.

El sistema empleado es un equipo EyeTribe de $60 \mathrm{~Hz}$ de tasa de muestreo con una precisión media de 0,5 grados de ángulo visual y una resolución espacial de $0,1^{\circ}$. Este equipo tiene una latencia de 20 milisegundos y admite 16 puntos para el proceso de calibración. Es apto para la investigación comercial, pues permite a los sujetos movimientos de hasta 75 centímetros en ángulos horizontales y verticales. El software experimental fue el OGAMA, es de libre uso y abierto, con registros científicos previos que validan su uso (Popelka, Stachoň, Šašinka, \& Doležalová, 2016; Voßkühler, Nordmeier, Kuchinke, \& Jacobs, 2008).

La secuencia experimental fue la siguiente. Primero los sujetos son informados y se recaba su consentimiento. Tras lo cual, se acomodan y se inicia el proceso de calibrado mediante la fijación y desplazamiento de la mirada sobre 16 puntos. Cada estímulo es mostrado durante ocho segundos con intervalo de una imagen negra de duración un segundo siguiendo el procedimiento de estudios previos (Gülçay \& Cangöz, 2016). Posteriormente, el sujeto es sometido a una encuesta para recabar sus datos sociodemográficos y de recuerdo.

\section{RESULTADOS}

La ceguera al patrocinador se analiza mediante un análisis descriptivo de la atención respecto del tiempo completo de fijación (CFT), tiempo hasta la primera fijación (TFF), y número de fijaciones (NF). Los datos sobre recuerdo de la marca completan el análisis. Con el fin de determinar las diferencias en cuanto al sexo se realiza un análisis de la varianza.

La Tabla 2 muestra en detalle los indicadores de fijación para el área de interés (AOI) fijada sobre la marca patrocinadora en el cartel. Para el estímulo B y D, cuando la marca patrocinadora está fuera del área de acción, la media del número de fijaciones es inferior o igual a 0,5 . Esto indica que la mitad de los sujetos no miraron nunca al patrocinador porque la media no llega a la unidad. Sin embargo, para los estímulos A y C, la media es superior a la unidad. Si se calcula el NF para el total de estímulos y disciplinas, se obtiene que: el porcentaje de sujetos que vieron la marca en alguno de los carteles es del $18 \%$, el porcentaje de sujetos que no vieron la marca en alguno de los carteles es del $52 \%$ y, por último, el porcentaje de sujetos que no vieron la marca en ninguno de los carteles es del 6,1\%. Además, del tiempo total de duración de la exposición (800oms), los patrocinadores recibieron una media de tan solo 332,6 milisegundos, el 4,16\%.

Tabla 2. Puntuaciones medias (M), desviación estándar (SD) y normalidad (N) para los indicadores de atención a la marca

\begin{tabular}{c|c|c|c|c|c|c|r|r|r}
\hline Tipo & \multicolumn{3}{|c|}{ CF } & \multicolumn{3}{c}{ TFF } & \multicolumn{3}{c}{ NF } \\
\hline & M & SD & N & M & SD & N & M SD & N \\
\hline B & 129 & 151,4 &, $8^{\star}$ & 2022,1 & 1828,2 &, $8^{*}$ &, 5 &, 5 &, $8^{*}$ \\
\hline D & 142,8 & 224,3 &, $6^{*}$ & 1341,1 & 1395,8 &, $8^{*}$ &, 4 &, 6 &, $7^{*}$ \\
\hline A & 526,9 & 321,3 &, 96 & 1845,1 & 1144,9 &, 94 & 1,3 &, 8 &, 91 \\
\hline C & 531,7 & 373,4 &, 9 & 1895,1 & 1237 &, 91 & 1,2 &, 7 &, 92 \\
\hline
\end{tabular}

* $p<.05$

La Figura 1 representa un ejemplo del análisis del mapa de fijaciones con paleta de colores rainbow. El mapa de calor indica un color más intenso (rojo) según el algoritmo matemático calcula la distribución normal de todas las fijaciones en función de su longitud. Un análisis exploratorio revela que los puntos con mayor fijación fueron la cara del jugador, la fecha, y el nombre del evento para el cartel del tenis. La torre de comunicaciones, el nombre del evento, y la fecha y el lugar de celebración para el evento de vela.

Posteriormente se examina en la Figura 2 el scanpath, que muestra el historial de fijaciones a través de los carteles como resultado del reflejo en la pupila de la luz infrarroja. Los números dentro de los círculos representan el lugar en el historial de fijación, y el tamaño del círculo, su duración. El replay puede verse en video en la siguiente dirección: https:// goo.gl/j6usRo.

Un análisis comparativo respecto del emplazamiento muestra que el mensaje recibe significativamente más atención cuando está situado dentro de la zona de acción que cuando está situado fuera para el CFT F $(1,118)=50,68$, p $<, 000 ;$ y el NFF $(1,118)=28,43, p<, 000$. No hubo diferencias en cuanto a TFF. 
ARTículos | CEGUERA AL PATROCINADOR: APLICACIÓN A CARTELES DE EVENTOS DEPORTIVOS

Manuel Alonso dos Santos | Ferran Calabuig Moreno | Manuel Jesús Sánchez-Franco

Figura 1. Mapa de calor para dos carteles de ejemplo del estímulo B

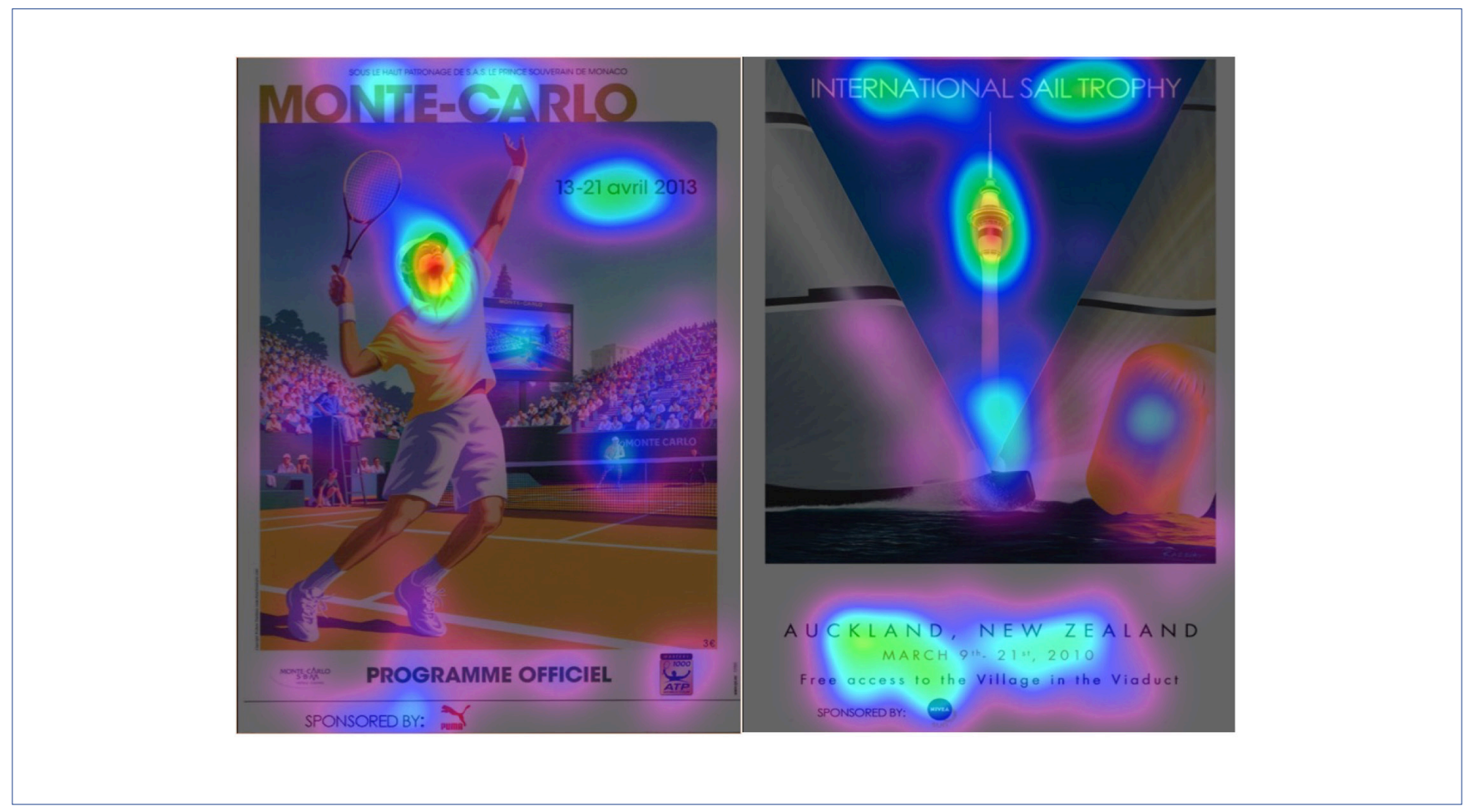

Fuente: OGAMA software.

Figura 2. Scanpath para los dos carteles de ejemplo del estímulo B
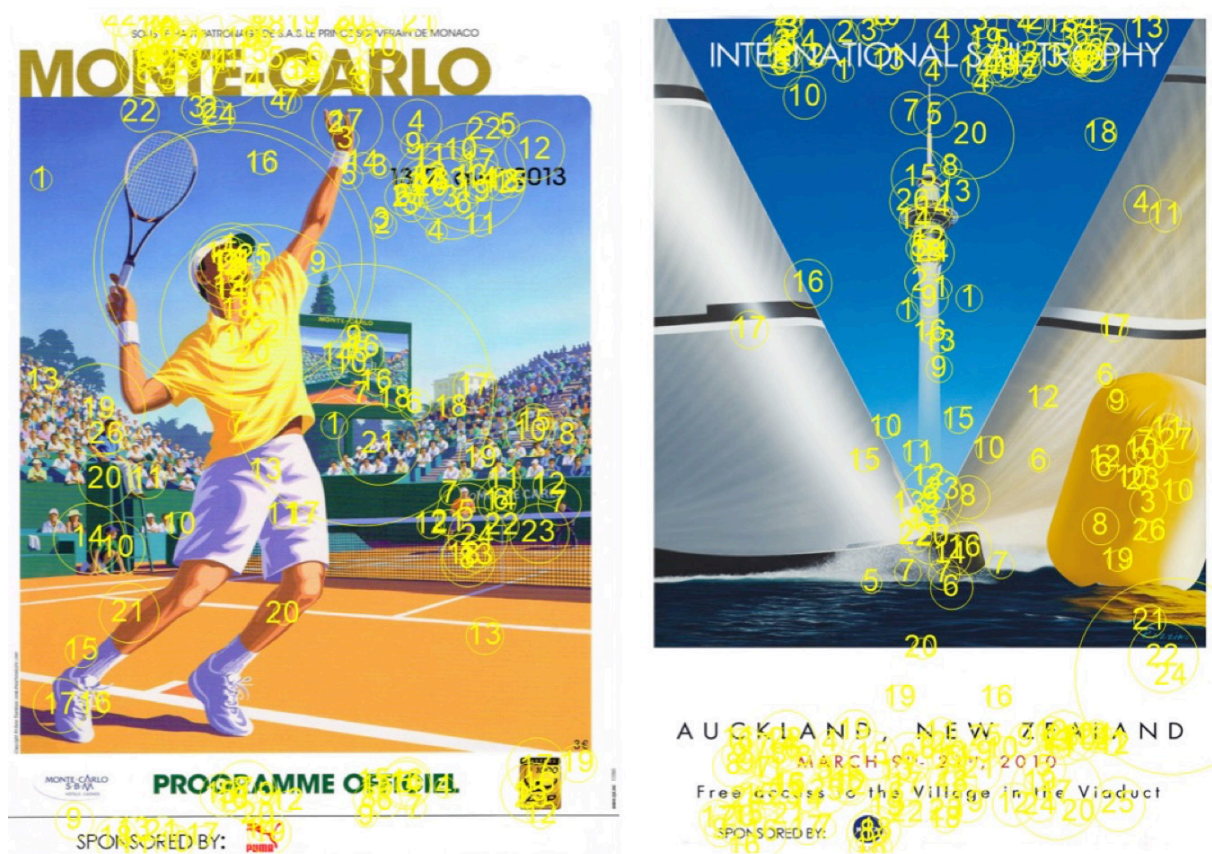

Fuente: OGAMA software. 
La Tabla 3 muestra la tasa de recuerdo espontáneo por sujetos. Los estímulos que mayor tasa de recuerdo presentan son los estímulos A y C. Estos estímulos presentan un patrocinador congruente e incongruente respectivamente, pero ambos dentro de la zona de acción. Lo que parece anunciar que los patrocinadores emplazados dentro tienen mayores tasas de recuerdo. Esto concuerda con el análisis de fijaciones en cuanto al CFT y NF realizado anteriormente.

La Figura 3 muestra las diferencias en la tasa de recuerdo en función del indicador CFT, siendo esta significativa $F(3,119)$ $=14,05, p<, 001$. También existen diferencias significativas para el resto de los indicadores, $\operatorname{TFF} F(3,118)=8,05, p<, 001$, y el $\mathrm{NFF}(3,119)=13,96, \mathrm{p}<, 001$. Estos resultados indican que las mayores tasas de recuerdo están relacionadas con mayores frecuencias en fijaciones, tiempo total de la fijación y tiempo hasta la primera fijación, estando esta última inversamente relacionada. Comparando la tasa de recuerdo empleando todos los pares según el método de Tukey-Kramer HSD obtenemos diferencias significativas entre todos los pares, excepto para el par 2-1. Lo que ciertamente ratifica tres niveles de recuerdo: 0 marcas, 1 y 2 marcas y 3 marcas.

\section{Tabla 3. Tasa de recuerdo del patrocinador $\mathbf{N}(\%)$}

\begin{tabular}{c|c|c|c|c}
\hline Estímulo & o aciertos & $\mathbf{1}$ acierto & $\mathbf{2}$ aciertos & 3 aciertos \\
\hline A & $7(23)$ & $15(50)$ & $5(17)$ & $3(10)$ \\
\hline B & $14(47)$ & $7(23)$ & $7(23)$ & $2(7)$ \\
\hline C & $7(23)$ & $14(47)$ & $6(20)$ & $3(10)$ \\
\hline D & $12(40)$ & $8(27)$ & $8(27)$ & $2(7)$ \\
\hline
\end{tabular}

Nota: $(n=30)$ por estímulo, cada estímulo tiene tres categorías deportivas y cada categoría un patrocinador.

Figura 3. Análisis del recuerdo y el indicador de fijación CFT

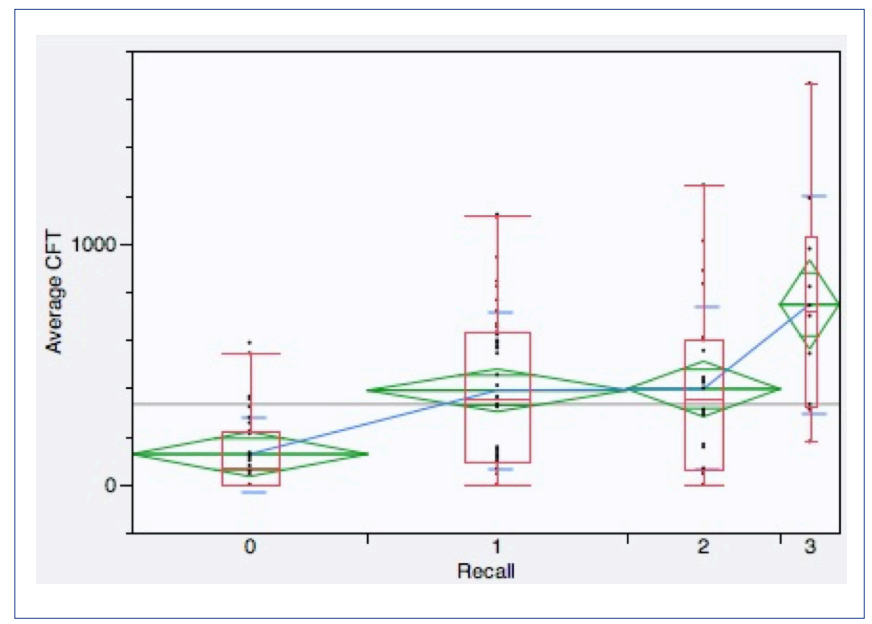

Nota: Las líneas rojas describen el diagrama de caja, la línea azul conecta los promedios, las líneas azules horizontales representan la desviación estándar y los rombos verdes representan los promedios y los intervalos de confianza.
Respecto del análisis del recuerdo y las fijaciones en función del sexo del sujeto, los resultados indican que no existe relación ni asociación entre el CFT (Figura 4), NF y TFF y el sexo, así como entre la tasa de recuerdo y el sexo. Resultados similares de atención al banner fueron encontrados por HernándezMéndez y Muñoz-Leiva (2015) al igual que Drèze y Hussherr (2003) y Barreto (2013).

\section{Figura 4. Análisis del sexo del sujeto y el indicador de fijación CFT}

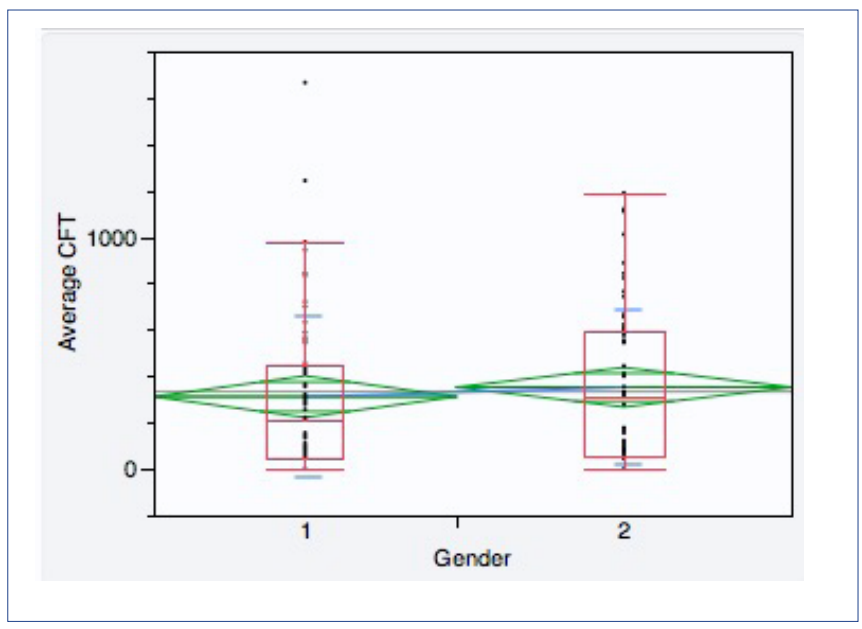

Nota: Las líneas rojas describen el diagrama de caja, la línea azul conecta los promedios, las líneas azules horizontales representan la desviación estándar y los rombos verdes representan los promedios y los intervalos de confianza.

\section{CONCLUSIONES Y RECOMENDACIONES}

El patrocinio deportivo es un área de estudio con significativa repercusión en el mundo académico (Meenaghan, 2013; Prendergast et al., 2016). Sin embargo, algunos medios de comunicación del patrocinio no han sido suficientemente abordados. En este sentido, el cartel deportivo ha recibido escasa atención en la literatura académica a pesar de sus ventajas, destacando su bajo coste, versatilidad y rápida respuesta (Nysveen \& Breivik, 2005).

El objetivo de esta investigación era medir el grado de eficacia de la cartelería deportiva y el patrocinio desde la óptica y aplicación del concepto de ceguera al banner. Por ello, hemos propuesto medir la atención prestada al patrocinador mediante indicadores de atención ocular y recuerdo. Además de segmentar la muestra para testar diferencias entre sexos. El diseño experimental constó de 12 estímulos de tres disciplinas deportivas (vela, F1 y tenis) y cuatro grupos de diez sujetos cada uno, aleatorizados y balanceados en función de la edad y el sexo del sujeto. Para dotar de mayor validez al experimento, los estímulos se dividieron en congruentes y no congruentes, 
con dos tipos de emplazamiento (dentro y fuera de la zona de acción).

Los resultados indican que sí existe ceguera al patrocinador. Acuñamos el término ceguera al patrocinador para referirnos a la tendencia de los sujetos de evitar procesar y prestar atención a los patrocinadores que se exponen en un cartel deportivo. Hasta donde alcanza nuestro conocimiento no existe literatura acerca de este concepto. Existe ceguera al patrocinador porque los indicadores de fijación revelan que tan solo el 4,16\% del tiempo de fijación total del cartel es dedicado al patrocinador. Un tiempo ligeramente inferior a la atención al patrocinador en la investigación de Breuer y Rumpf (2012), salvando las diferencias conceptuales y metodológicas por falta de estudios en el área con qué comparar. Más de la mitad de los sujetos del experimento no prestaron atención a alguno de los patrocinadores de los tres carteles. Estos resultados se sustentan posteriormente mediante un análisis de los mapas de calor y scanpath, donde se observa que los sujetos dedican cortos espacios de tiempo al procesamiento del patrocinador. La falta de atención podría deberse a la sobrecarga de información presente en el cartel (Tangmanee, 2016). No obstante, la colocación del patrocinador en la parte baja del cartel también podría evidenciar estos resultados. Según Breuer y Rumpf (2012) y con base en la teoría de la sobrecarga, la atención puede predecirse en función de la colocación de la marca del patrocinador en el medio publicitario y de otras variables de exposición. El tiempo que es absorbido por la información del evento puede ser restado del tiempo de procesamiento de la marca del patrocinador. Por lo tanto, es necesario que futuras investigaciones alternen la posición de los patrocinadores, y no solo en la zona de acción. Así mismo, es necesario trasladar estas investigaciones al ámbito profesional. Es necesario que el patrocinador conozca la atención que ha recibido su marca, no solo el número de veces que aparece en pantalla o monitores. Por ejemplo, entre las últimas tendencias se están desarrollando algoritmos capaces de predecir y valorar la exposición de los patrocinios en redes sociales (visite, por ejemplo, blinkfire.com), pero sin valorar si ciertamente la marca del patrocinador fue observada y cuánta atención recibió.

Se pudo demostrar que los patrocinadores situados en la zona de acción reciben más atención que el resto de los patrocinadores. Pero no pudimos demostrar que la atención varíe en función de la congruencia. La literatura tiende a afirmar que cuanto mayor congruencia, mejor procesamiento de la información (Cornwell et al., 2006). Sin embargo, diversos estudios apuntan en el sentido contrario (Alonso Dos Santos \& Calabuig, 2018; Tribou, 2011). En cualquier caso, nuestros resultados no pudieron inclinar la discusión hacia ningún sentido.
Así mismo, aunque no pudimos demostrarlo, la literatura académica muestra que la eficacia del emplazamiento puede variar en función de su congruencia (DeLorme \& Reid, 1999). Pero, este último resultado podría estar influenciado por la ceguera al patrocinador. El sujeto podría no tener el tiempo suficiente para procesar la congruencia o no del mensaje. Esto sugiere que el emplazamiento en la zona de acción debería ser únicamente destinado al patrocinador principal, que obtiene un mayor rendimiento en cuanto a atención a su marca.

Por otro lado, se obtuvieron datos del recuerdo sin asistencia de la marca mediante pregunta posterior al estímulo. De las tres marcas mostradas en el experimento, tan solo entre un $8 \%$ y un $10 \%$ de los sujetos fueron capaces de recordar dos tercios de las marcas. Lo que representa un porcentaje relativamente bajo (Tangmanee, 2016) pero en concordancia con estudios previos en entornos en línea (Hamborg, Bruns, Ollermann, \& Kaspar, 2012). Esta comparación debe realizarse con cautela, pues los métodos, entornos y áreas son disímiles. En el ámbito deportivo, las tasas de recuerdo también son difícilmente comparables. Siguiendo a Carrillat, d'Astous, Bellavance y Eid (2015), las investigaciones han sido muy heterogéneas en cuanto a muestras, eventos, patrocinadores y metodologías. Carrillat et al. (2015) muestran la disparidad en éxito de recuerdo entre diferentes investigaciones, situándose la tasa de recuerdo entre el 9,25\% y el 68\% (Nicholls, Roslow, \& Dublish, 1999; Quester, 1997). Una brecha demasiado extensa para poder realizar una comparación oportuna o poder fijar un límite recomendado, pero inferior a las tasas habituales de recuerdo mostradas en investigaciones previas. Algunas de ellas situadas en torno al $63 \%$ (Barros et al., 2007), superiores al 40\% (Cornwell, Humphreys, Maguire, Weeks, \& Tellegen, 2006) o al 20\% (Breuer \& Rumpf, 2012). Es importante mantener presente la relación entre el recuerdo y el resultado estratégico de la inversión de patrocinio, pues constituye uno de los indicadores más valorados por las empresas como signo de la eficacia de su inversión (DeGaris, Kwak, \& McDaniel, 2017).

Estos resultados conllevan varias recomendaciones dirigidas a los patrocinadores deportivos. En primer lugar, es mejor posicionarse dentro de la zona de acción de la comunicación en términos de atención. Aunque no hemos podido comprobar la rentabilidad económica que podría derivarse de un posible contrato de patrocinador principal que conlleve este derecho. En segundo lugar, la posible ceguera al patrocinador podría verse modificada con cambios de situación (Resnick \& Albert, 2014), animación de los carteles digitales (Hernández-Méndez \& Muñoz-Leiva, 2015) o congruencia con el entorno o patrocinado (Rieger et al., 2015). En cualquier caso, el sexo no parece tener un efecto sobre la 
atención, lo que indica que estrategias de segmentación de la comunicación en función del sexo no parecen tener efecto sobre el patrocinador. En último lugar, es recomendable que los patrocinios con mayor inversión económica puedan testar su eficacia por medio de una metodología fiable. El uso del seguimiento ocular, como se ha demostrado en este trabajo, puede ser un método fiable de medición de la eficacia de la comunicación en patrocinio. Además, tal y como señalan Breuer y Rumpf (2012), este tipo de investigaciones evidencian el proceso cognitivo de entrada y salida de información en el consumidor, en la medida en que somos capaces de analizar y precisar cuánta información procesó el sujeto y cómo la información ha sido resuelta. Sin embargo, el sistema de procesamiento y el procesamiento inconsciente de la marca y su asociación con otros conceptos no ha sido observado (Cornwell et al., 2006), lo que constituye en sí una línea de investigación que aún es necesario investigar.

Es importante asumir que la muestra empleada en esta investigación no es representativa de la población, particularmente por el uso de estudiantes universitarios. Como asimismo, las categorías deportivas se escogieron por conveniencia. Sería interesante, de cara a futuras investigaciones, replicar este experimento en situaciones reales, pues el contexto del laboratorio constituye una limitación a tener en cuenta. Otras variables podrían incluirse para medir los efectos moderadores que pudieran alterar la eficacia de la comunicación en patrocinio. La implicación con el deporte y el equipo o atleta podría ser una variable a tener en cuenta para su estudio. El medio de exposición, así como la repetición del mensaje, podría también significar avances significativos en la comprensión de la eficacia del patrocinio.

\section{REFERENCIAS}

Alonso Dos Santos, M. (Ed.). (2014). Strategies in sports marketing: Technologies and emerging trends. Hershey, USA: IGI Global.

Alonso Dos Santos, M., \& Calabuig, F. (2018). Assessing the effectiveness of sponsorship messaging: Measuring the impact of congruence through electroencephalogram. International Journal of Sports Marketing and Sponsorship, 19(1), 25-40. doi:10.1108/ IJSMS-09-2016-0067

Alonso Dos Santos, M., Calabuig, F., Rejón Guardia, F., \& PérezCampos, C. (2016). Influence of the virtual brand community in sports sponsorship. Psychology \& Marketing, 33(12), 1091-1097. doi:10.1002/mar.20943

Alonso Dos Santos, M., \& Pérez-Campos, C. (2015). ¿Influyen las emociones en la intención de asistir a un evento deportivo? Diferencias de género. Journal of Sports Economics \& Management, 5(1), 2-16.

Amorim, J. G. B., \& Almeida, V. M. C. (2015). The effect of simultaneous sponsorship of rival football teams. BAR-Brazilian Administration Review, 12(1), 63-87. doi:10.1590/1807-7692bar2015140059
Barreto, A. M. (2013). Do users look at banner ads on Facebook? Journal of Research in Interactive Marketing, 7(2), 119-139. doi:10.1108/JRIMMar-2012-0013

Barros, C. P., Barros, C. de, Santos, A., \& Chadwick, S. (2007). Sponsorship brand recall at the Euro 2004 Soccer Tournament. Sport Marketing Quarterly, 16(3), 161-170.

Bennett, R. (1999). Sports sponsorship, spectator recall and false consensus. European Journal of Marketing, 33(3/4), 291-313. doi:10.1108/03090569910253071

Boronczyk, F., Rumpf, C., \& Breuer, C. (2018). Determinants of viewer attention in concurrent event sponsorship. International Journal of Sports Marketing and Sponsorship, 19(1), 11-24. doi:10.1108/ IJSMS-09-2016-0063

Breuer, C., \& Rumpf, C. (2012). The viewer's reception and processing of sponsorship information in sport telecasts. Journal of Sport Management, 26(6), 521-531. doi:10.1123/jsm.26.6.521

Breuer, C., \& Rumpf, C. (2015). The impact of color and animation on sports viewers' attention to televised sponsorship signage. Journal of Sport Management, 29(2), 170-183. doi:10.1123/JSM.2013-0280

Carrillat, F. A., d'Astous, A., Bellavance, F., \& Eid, F. (2015). On "being there": A comparison of the effectiveness of sporting event sponsorship among direct and indirect audiences. European Journal of Marketing, 49(3/4), 621-642. doi:10.1108/EJM-03-2013-0156

Choi, Y. S., \& Yoh, T. (2011). Exploring the effect of communication channels on sponsorship effectiveness: A case study of Super Bowl XLII. International Journal of Sport Management \& Marketing, 9(1/2), 75-93. doi:10.1504/IJSMM.2011.040258

Cianfrone, B. A. (2007). The influence of motives and consumption of sport video games on sponsorship effectiveness (Ph.D. thesis). Disponible en ProQuest Dissertations and Theses database.

Close, A. G., Lacey, R., \& Cornwell, T. B. (2015). Visual processing and need for cognition can enhance event-sponsorship outcomes. Journal of Advertising Research, 55(2), 206-215. doi:10.2501/JAR-552-206-215

Conradie, M. (2011). The 2010 FIFA World Cup as a commercial platform in print advertising. African Identities, 9(3), 291-305. doi:10.1080/14 725843.2011 .591229

Cornwell, T. B., Humphreys, M. S., Maguire, A. M., Weeks, C. S., \& Tellegen, C. L. (2006). Sponsorship-linked marketing: The role of articulation in memory. Journal of Consumer Research, 33(3), 312-321. doi:10.1086/508436

Cornwell, T. B., Lipp, O. V., \& Purkis, H. (2006). Examination of affective responses to images in sponsorship-linked marketing. Journal of Global Sport Management, 1(3-4), 110-128. doi:10.1080/24704067. 2016.1240947

Dardis, F. E. (2009). Attenuating the negative effects of perceived incongruence in sponsorship: How message repetition can enhance evaluations of an "incongruent" sponsor. Journal of Promotion Management, 15(1-2), 36-56. doi:10.1080/10496490902837759

d'Astous, A., \& Séguin, N. (1999). Consumer reactions to product placement strategies in television sponsorship. European Journal of Marketing, 33(9/10), 896-910. doi:10.1108/03090569910285832

DeGaris, L., Kwak, D. H., \& McDaniel, S. R. (2017). Modeling the effects of sponsorship-linked marketing: When does memory matter? Journal of Promotion Management, 23(2), 320-339. doi:10.1080/10 496491.2016.1267678 
DeLorme, D. E., \& Reid, L. N. (1999). Moviegoers' experiences and interpretations of brands in films revisited. Journal of Advertising, 28(2), 71-95. doi:10.1080/00913367.1999.10673584

Drèze, X., \& Hussherr, F.-X. (2003). Internet advertising: Is anybody watching? Journal of Interactive Marketing, 17(4), 8-23. doi:10.1002/ dir.10063

Dudzik, T., \& Gröppel-Klein, A. (2005). The efficiency of integrated sponsorship advertising. International Journal of Sports Marketing and Sponsorship, 7(1), 51-61. doi:10.1108/IJSMS-07-01-2005-Boo9

Funk, D. C., Toohey, K., \& Bruun, T. (2008). International sport event participation: Prior sport involvement, destination image, and travel motives. European Sport Management Quarterly, 7(3), 227-248. doi:10.1080/16184740701511011

Goodrich, K. (2014). The gender gap: Brain-processing differences between the sexes shape attitudes about online advertising. Journal of Advertising Research, 54(1), 32-43. doi:10.2501/JAR-54-1-032-043

Grohs, R. (2015). Drivers of brand image improvement in sports-event sponsorship. International Journal of Advertising, 35(3), 391-420. do $\mathrm{i}: 10.1080 / 02650487.2015 .1083070$

Gross, P., \& Wiedmann, K.-P. (2015). The vigor of a disregarded ally in sponsorship: Brand image transfer effects arising from a cosponsor. Psychology \& Marketing, 32(11), 1079-1097. doi:10.1002/mar.20848

Gülçay, Ç., \& Cangöz, B. (2016). Effects of emotion and perspective on remembering events: An eye-tracking study. Journal of Eye Movement Research, 9(2), 1-19. doi:10.16910/jemr.9.2.4

Hamborg, K.-C., Bruns, M., Ollermann, F., \& Kaspar, K. (2012). The effect of banner animation on fixation behavior and recall performance in search tasks. Computers in Human Behavior, 28(2), 576-582. doi:10.1016/j.chb.2011.11.003

Hernández-Méndez, J., \& Muñoz-Leiva, F. (2015). What type of online advertising is most effective for eTourism 2.0? An eye tracking study based on the characteristics of tourists. Computers in Human Behavior, 50, 618-625. doi:10.1016/j.chb.2015.03.017

Hsieh, Y.-C., \& Chen, K.-H. (2011). How different information types affect viewer's attention on internet advertising. Computers in Human Behavior, 27(2), 935-945. doi:10.1016/j.chb.2010.11.019

Keller, K. L. (1993). Conceptualizing, measuring, and managing customer-based brand equity. Journal of Marketing, 57(1), 1-22. doi:10.2307/1252054

Kelly, S., Coote, L., Cornwell, T. B., \& McAlister, A. (2017). Mellowing skeptical consumers: An examination of sponsorship-linked advertising. International Journal of Sport Communication, 10(1), 58 84. doi:10.1123/ijsc.2016-0099

Kinney, L., McDaniel, S. R., \& DeGaris, L. (2008). Demographic and psychographic variables predicting NASCAR sponsor brand recall. International Journal of Sports Marketing \& Sponsorship, 9(3), 11-21. doi:10.1108/IJSMS-09-03-2008-B005

Ko, Y. J., Kim, M. K., Kim, Y. K., Lee, J.-H., \& Cattani, K. (2010). Consumer satisfaction and event quality perception: A case of US Open Taekwondo Championship. Event Management, 14(3), 205-214. doi:1 $0.3727 / 152599510 X_{12825895093597}$

Kwon, H. H., \& Armstrong, K. L. (2002). Factors influencing impulse buying of sport team licensed merchandise. Sport Marketing Quarterly, 11(3), 151-163.
Lardinoit, T., \& Derbaix, C. (2001). Sponsorship and recall of sponsors. Psychology \& Marketing, 18(2), 167-190. doi:10.1002/15206793(200102)18:2«167::AID-MAR1004/3.0.CO;2-I

Lee, L.-Y. (2008). Perception of event-sponsor personality congruence, event-sponsor fit, and sponsorship outcomes: An international sports event in Taiwan (D.B.A.). Ann Arbor, United States. Retrieved from http://search.proquest.com/docview/304818223/abstract/ $\mathrm{E}_{12} \mathrm{C}_{7} \mathrm{E} 9 \mathrm{BEA} 2540 \mathrm{~A} 5 \mathrm{PQ} / 3$ ?accountid $=14542$

Lee, S. (2010). Influence of brand exposure frequency and sport involvement on sport sponsorship effects: An examination of mere exposure (Ph.D., Indiana University, Indiana, USA). Obtenido de http://search.proquest.com/docview/594581065/ abstract $/ 13884$ CCC8B $757301880 / 5$ ?accountid $=14542$

Liu, X., Hu, M. Y., \& Grimm, P. E. (2010). Affect transfer in brand extensions: The role of expectancy and relevancy. Journal of Product \& Brand Management, 19(5), 317-326. doi:10.1108/10610421011068559

Madrigal, R. (2001). Social identity effects in a belief-attitudeintentions hierarchy: Implications for corporate sponsorship. Psychology \& Marketing, 18(2), 145-165. doi:10.1002/15206793(200102)18:2〈145::AID-MAR1003'3.0.CO;2-T

Maxwell, H., \& Lough, N. (2009). Signage vs. no signage: An analysis of sponsorship recognition in women's college basketball. Sport Marketing Quarterly, 18(4), 188-198.

McDaniel, S., \& Kinney, L. (1999). Audience characteristics and event sponsorship response: The potential influence of demographics, personal interests and values on brand awareness and brand image. International Journal of Sports Marketing \& Sponsorship, 1(2), 15-35. doi:10.1108/IJSMS-01-02-1999-Boo3

McMahan, C., Hovland, R., \& McMillan, S. (2009). Online marketing communications: Exploring online consumer behavior by examining gender differences and interactivity within internet Advertising. Journal of Interactive Advertising, 10(1), 61-76. doi:10.1080/152520 19.2009.10722163

Meenaghan, T. (2001). Understanding sponsorship effects. Psychology \& Marketing, 18(2), 95-122. doi:10.1002/15206793(200102)18:2〈95::AID-MAR1001〉3.0.CO;2-H

Meenaghan, T. (2013). Measuring sponsorship performance: Challenge and direction. Psychology \& Marketing, 30(5), 385-393. doi:10.1002/ mar.20613

Meenaghan, T., \& Shipley, D. (1999). Media effect in commercial sponsorship. European Journal of Marketing, 33(3/4), 328-348. doi:10.1108/03090569910253170

Menefee, W. C., \& Casper, J. M. (2011). Professional basketball fans in China: A comparison of National Basketball Association and Chinese Basketball Association team identification. International Journal of Sport Management \& Marketing, 9(3/4), 185-200. doi:10.1504/ IJSMM.2011.041571

Meyers-Levy, J. (1988). The influence of sex roles on judgment. Journal of Consumer Research, 14(4), 522-530. doi:10.1086/209133

Muñiz, C., Rodríguez, P., \& Suárez, M. J. (2014). Sports and cultural habits by gender: An application using count data models. Economic Modelling, 36, 288-297. doi:10.1016/j.econmod.2013.09.053

Muñoz-Leiva, F., Hernández-Méndez, J., Liébana-Cabanillas, F. J., \& Marchitto, M. (2016). Analysis of advertising effectiveness and usability in Travel 2.0 tools. An experimental study through eyetracking technique. Tourism \& Management Studies, 12(2), 7-17. 
Nicholls, J. A. F., Roslow, S., \& Dublish, S. (1999). Brand recall and brand preference at sponsored golf and tennis tournaments. European Journal of Marketing, 33(3/4), 365-387. doi:10.1108/03090569910253198

Nysveen, H., \& Breivik, E. (2005). The influence of media on advertising effectiveness a comparison of internet, posters and radio. International Journal of Market Research, 47(4), 381-404. doi:10.1177/147078530504700405

Ortiz-Chaves, L., Martínez-de-Pisón, E., Cancela-López-Carrión, G. Gonçalves-de-Vasconcellos, J., \& Marcos, M.-C. (2014). AdWords, imágenes y ceguera a los banners. Un estudio con eye tracking. $E l$ Profesional de la Información, 23(3), 279-287.

Papadimitriou, D., Kaplanidou, K. K., \& Papacharalampous, N. (2016). Sport event-sponsor fit and its effects on sponsor purchase intentions: A non-consumer perspective among athletes, volunteers and spectators. Journal of Business \& Industrial Marketing, 31(2), 247-259. doi:10.1108/JBIM-09-2014-0187

Pitts, B. G., \& Slattery, J. (2004). An examination of the effects of time on sponsorship awareness levels. Sport Marketing Quarterly, 13(1), 43-54.

Pope, N. K. L., \& Voges, K. E. (1999). Sponsorship and image: A replication and extension. Journal of Marketing Communications, 5(1), 17-28. doi:10.1080/135272699345716

Popelka, S., Stachoň, Z., Šašinka, Č., \& Doležalová, J. (2016). EyeTribe tracker data accuracy evaluation and its interconnection with hypothesis software for cartographic purposes. Computational Intelligence and Neuroscience, 2016 (Article ID 9172506). doi:10.1155/2016/9172506

Porta, M., Ravarelli, A., \& Spaghi, F. (2013). Online newspapers and ad banners: An eye tracking study on the effects of congruity. Online Information Review, 37(3), 405-423. doi:10.1108/0IR-01-2012-0001

Prendergast, G., Paliwal, A., \& Mazodier, M. (2016). The hidden factors behind sponsorship and image transfer. Journal of Advertising Research, 56(2), 132-135. doi:10.2501/JAR-2016-026

Quester, P. G. (1997). Awareness as a measure of sponsorship effectiveness: The Adelaide Formula One Grand Prix and evidence of incidental ambush effects. Journal of Marketing Communications, 3(1), 1-20. doi:10.1080/135272697346014

Rath, S., \& Mousumi Singha Mohapatra. (2013). Brand recognition and advertising effectiveness: Study of youth responses for select print advertisements. Journal of Contemporary Management Research, $8(2), 82-88$.

Redker, C., Gibson, B., \& Zimmerman, I. (2013). Liking of movie genre alters the effectiveness of background product placements. Basic and Applied Social Psychology, 35(3), 249-255. doi:10.1080/019735 33.2013 .785400

Resnick, M., \& Albert, W. (2014). The impact of advertising location and user task on the emergence of banner ad blindness: An eye-tracking study. International Journal of Human-Computer Interaction, 30(3), 206-219. doi:10.1080/10447318.2013.847762

Resnick, M. L., \& Albert, W. (2016). The influences of design esthetic, site relevancy and task relevancy on attention to banner advertising. Interacting with Computers, 28(5), 680-694. doi:10.1093/iwc/iwv042

Rieger, D., Bartz, F., \& Bente, G. (2015). Reintegrating the ad: Effects of context congruency banner advertising in hybrid media. Journal of Media Psychology, 27(2), 64-77. doi:10.1027/1864-1105/a0o0131
Rumpf, C., \& Breuer, C. (2014). Predicting the attentional effect of sport sponsorship information as an innovative evaluation approach. In Alonso Dos Santos, M. (Ed.), Strategies in sports marketing: Technologies and emerging trends (pp. 238-254). Hershey, USA: IGI Global.

Russell, J. A. (1980). A circumplex model of affect. Journal of Personality and Social Psychology, 39(6), 1161-1178. doi:10.1037/hoo77714

Simola, J., Kivikangas, M., Kuisma, J., \& Krause, C. M. (2013). Attention and memory for newspaper advertisements: Effects of ad-editorial congruency and location. Applied Cognitive Psychology, 27(4), 429442. doi:10.1002/acp. 2918

Speed, R., \& Thompson, P. (2000). Determinants of sports sponsorship response. Academy of Marketing Science Journal, 28(2), 226-238. doi:10.1177/0092070300282004

Stipp, H., \& Nicholas, P. (1996). Modeling the impact of Olympic sponsorship on corporate image. Journal of Advertising Research, 36(4), 22-28.

Tangmanee, C. (2016). Fixation and recall of YouTube ad banners: An eye-tracking study. International Journal of Electronic Commerce Studies, 7(1), 49-75. doi:10.7903/ijecs.1404

Tipps, S. W., Berger, P. D., \& Weinberg, B. D. (2006). The effect of media involvement on print advertising effectiveness. Journal of Promotion Management, 12(2), 53-75. doi:10.1300/J057V12no2_06

Tom, G., Nelson, C., Srzentic, T., \& King, R. (2007). Mere exposure and the endowment effect on consumer decision making. The Journal of Psychology, 141(2), 117-125. doi:10.3200/JRLP.141.2.117-126

Tribou, G. (2011). Sponsorship: Associating image attributes with specific sports and particular teams. International Journal of Sports Marketing \& Sponsorship, 12(2), 37-51. doi:10.1108/IJSMS-12-022011-Boo5

Uhrich, S., Koenigstorfer, J., \& Groeppel-Klein, A. (2014). Leveraging sponsorship with corporate social responsibility. Journal of Business Research, 67(9), 2023-2029. doi:10.1016/j.jbusres.2013.10.008

Valenzuela, L. F., Martínez, C. T., \& Yáñez, F. W. (2015). Influence of placement on explicit and implicit memory of college students. Comunicar, 22(44), 169-176. doi:10.3916/C44-2015-18

Voßkühler, A., Nordmeier, V., Kuchinke, L., \& Jacobs, A. M. (2008). OGAMA (Open Gaze and Mouse Analyzer): Open-source software designed to analyze eye and mouse movements in slideshow study designs. Behavior Research Methods, 40(4), 1150-1162. doi:10.3758/ BRM.40.4.1150

Wann, D. L., Melnick, M. J., Russell, G. W., \& Pease, D. G. (2001). Sport fans: The psychology and social impact of spectators. New York, NY: Routledge Press.

Wann, D. L., Waddill, P. J., \& Dunham, M. D. (2004). Using sex and gender role orientation to predict level of sport fandom. Journal of Sport Behavior, 27(4), 367-377.

Wells, W. D. (2000). Recognition, recall, and rating scales. Journal of Advertising Research, 40(6), 14-20. doi:10.2501/JAR-40-6-14-20

Zouharová, M., Zouhar, J., \& Smutný, Z. (2016). A MILP approach to the optimization of banner display strategy to tackle banner blindness. Central European Journal of Operations Research, 24(2), 473-488. doi:10.1007/s10100-015-0398-3 